EVS28

KINTEX, Korea, May 3-6, 2015

\title{
Thermal Model Developments for Electrified Vehicles
}

\author{
Namwook Kim ${ }^{1}$, Namdoo Kim ${ }^{1}$, Aymeric Rousseau ${ }^{1}$ \\ ${ }^{1}$ Argonne National Laboratory, 9700 S. Cass Ave, Lemont, IL60439, USA
}

\begin{abstract}
Argonne National Laboratory has analyzed the control behavior of advanced vehicles, such as hybrid electric vehicles (HEVs), plug-in hybrid electric vehicles (PHEVs), and battery electric vehicles (BEVs), to develop simulation models and to reproduce the performance of vehicles with simulation techniques. Since many of the novel and advanced studies about transportation technologies done at Argonne use these simulation techniques, they must be well-validated to conduct and support these studies. To improve its research ability, Argonne built a new testing facility that can test vehicles under different thermal conditions (e.g., $-7^{\circ} \mathrm{C}$ or $35^{\circ} \mathrm{C}$ ), and it has analyzed the controls and performance of several advanced vehicles under these conditions. Further, Argonne has used the analyzed results to develop thermal component models that reproduce the thermal behavior of the vehicles. A main reason to develop thermal models is that the thermal conditions have such a significantly large impact on vehicle performance, especially with regard to advanced vehicles like HEVs or PHEVs. For instance, engine and battery efficiencies must decrease at low temperatures since the battery might not be able to provide enough power if it is very cold. Moreover, the climate control system still has a great demand for additional energy under very cold weather conditions even if the engine is not operating at all. The test data obtained from Argonne's Advanced Powertrain Research Facility (APRF) are analyzed in order to understand the thermal impacts on controls and performance, and the thermal models are developed based on the analyzed results and validated with the test data. In comparative studies, the simulation models have been found to reproduce fuel consumption that is very close to the fuel consumption obtained from the tests.
\end{abstract}

Keywords: Hybrid Electric Vehicle, Thermal Model, Control, Dynamometer Test, Fuel Economy

\section{Introduction}

Electrified vehicles such as hybrid electric vehicles (HEVs), plug-in hybrid electric vehicles (PHEVs), and battery electric vehicles (BEVs) are able to save fossil fuels by optimizing the engine's operations, recuperating the braking energy, or using electric energy instead of the fuel. Simply stated, the electrified vehicles have an additional degree of freedom to manage energy by being able to split the power between the fuel and electricity, whereas a conventional vehicle only uses the fuel. One issue that arises from this additional capability is that the engine might not provide enough waste heat to warm up a passenger compartment or the powertrain components, including the engine itself. [1] Because of that, the electrified vehicles may suffer from the inefficient operation of the 
powertrain components under very cold ambient temperatures [2]-[3]. For instance, Figure 1 compares the energy consumption results for four types of electrified vehicles; they were obtained from chassis dynamometer tests done at different ambient room temperatures.

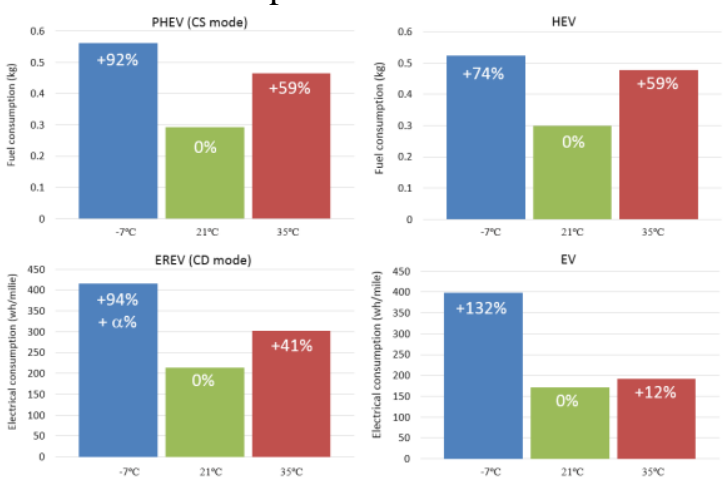

Figure 1. Energy consumption results obtained from chassis dynamometer tests.

The results show that electrified vehicles consume a lot of fuel or electricity in cold ambient temperature tests $\left(-7^{\circ} \mathrm{C}\right)$ : almost twice as much as they use in normal ambient temperature tests $\left(21^{\circ} \mathrm{C}\right)$. The increase in energy consumption is caused by the heat load demand from the passenger compartment or by the low efficiency of the engine's operations; the increase is so significantly large that the new test procedures published by U.S. Environmental Protection Agency (EPA) include additional tests under cold and hot ambient conditions for the fuel economy labels of vehicles produced in 2008 or later. For the same reason, developing thermal models for the electrified vehicles is also very important; the fuel efficiencies of the vehicles should include the impact of thermal conditions. Argonne National Laboratory has been developing a number of advanced vehicle models, including electrified vehicles, to analyze the impacts of new technologies on fuel efficiency and market penetration [4]-[6]. Also, the laboratory has been conducting various studies to improve fuel efficiency by optimizing the control algorithm or the vehicle parameters. In recent years, Argonne has been adding value by improving the model's fidelity, and one ongoing effort is to develop the vehicle's thermal management system [1]. This brief provides information about the entire process - conducting the tests, analyzing the test data, and developing the vehicle model - so that the process can be used in other studies.

\section{Control and \\ Performance Analysis}

To have well validated simulation models, it is also very important to have well qualified test results. Based on the test results, the performance of the vehicle components can be analyzed, and the control of the vehicle system can be also analyzed before being implemented into the simulation models.

\subsection{Chassis Dynamometer Tests}

Argonne built the Advanced Powertrain Research Facility (APRF), which has a chassis dynamometer in a thermal chamber, to be a testing facility that can evaluate the fuel consumption and emissions of advanced vehicles under different thermal conditions (Figure 2) [7], [8].

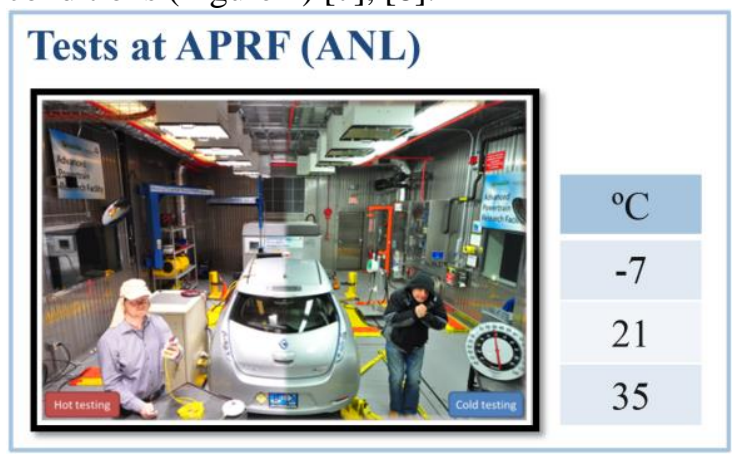

Figure 2. APRF in a thermal chamber

The facility is able to control room temperatures, so the vehicles can be tested to evaluate their performance under different ambient room temperatures like $-7^{\circ} \mathrm{C}$ or $35^{\circ} \mathrm{C}$. Additional instruments like solar lamps and fans are also implemented to mimic real world operating conditions. In the facility, Argonne has been testing many advanced vehicles, and the testing results are selectively available at the website [9], [10].

\subsection{Performance Analysis}

The main goal of the performance analysis for thermal model development is to determine the impact of temperature on a powertrain component's efficiency. Figures 3 and 4 show two examples for the performance analysis: Figure 3 is for the engine, and Figure 4 is for the battery. The test results for Figure 3 show that the engine's fuel consumption rate is changed mostly by the engine's speed and the engine's torque but is also significantly changed by the engine's coolant temperature. 


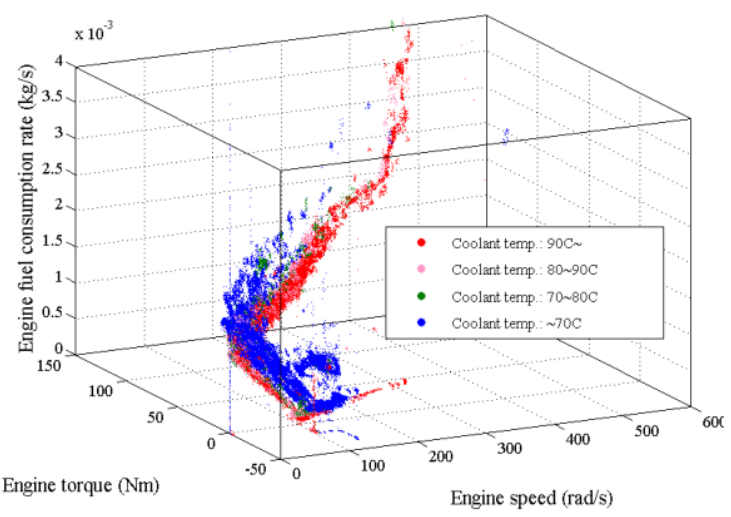

Figure 3 . The engine fuel consumption rate according to the engine coolant temperature

On the other hand, Figure 4 results show that the internal resistance, which affects the battery efficiency, also significantly changes according to the battery temperature.

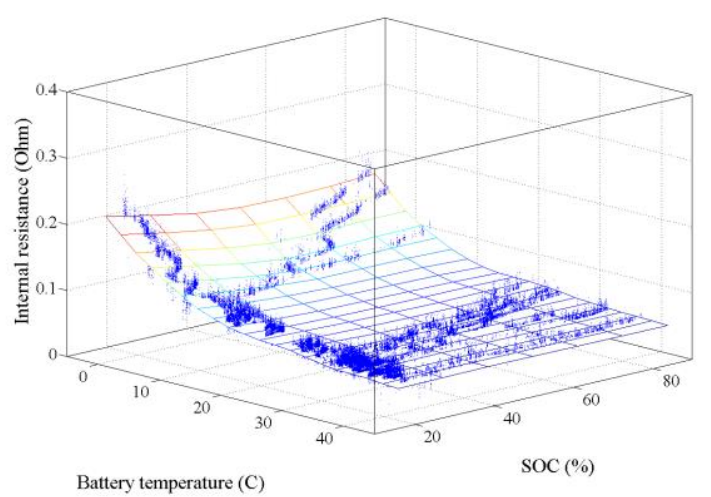

Figure 4 . The internal resistance of the battery obtained from test data

Based on the analysis of the test data, the performance of the powertrain components can be applied in the simulation models.

\subsection{Control Analysis}

The other important step in the analysis process is to analyze the control behavior of the real world vehicle. The goal of the vehicle model development is to evaluate the vehicle's performance in simulation environments, for which the control used in the real world vehicle should be well implemented in the control model. For instance, Figure 5 shows the engine on/off condition of a Toyota Prius HEV according to the vehicle speed and wheel torque demand, and Figure 6 shows that the on/off condition changes according to the thermal condition of the engine.

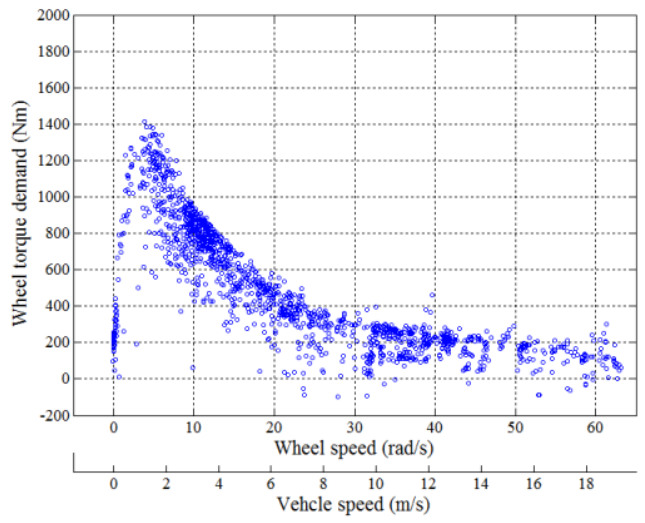

Figure 5. Engine on/off analysis based on test data
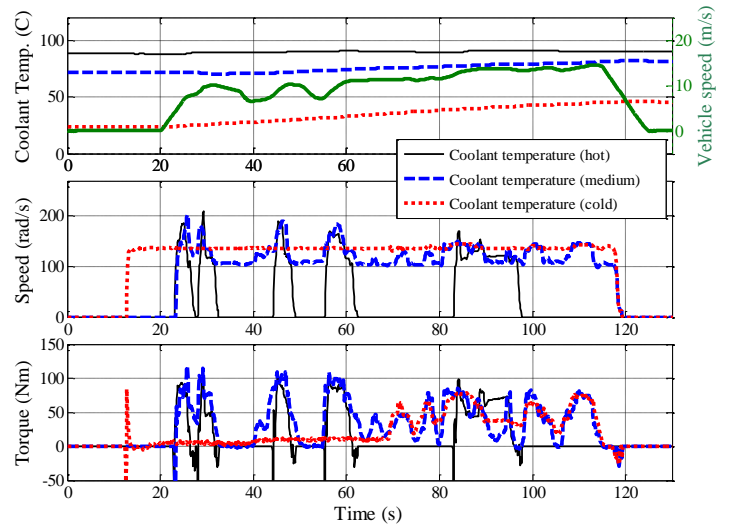

Figure 6 . The engine on/off condition as changed by the level of the engine coolant temperature

On the other hand, Figures 7 and 8 were obtained by analyzing the test data for the battery, which show that the output power of the battery is controlled by its State of Charge (SOC), and that battery power is limited according to the battery temperature.

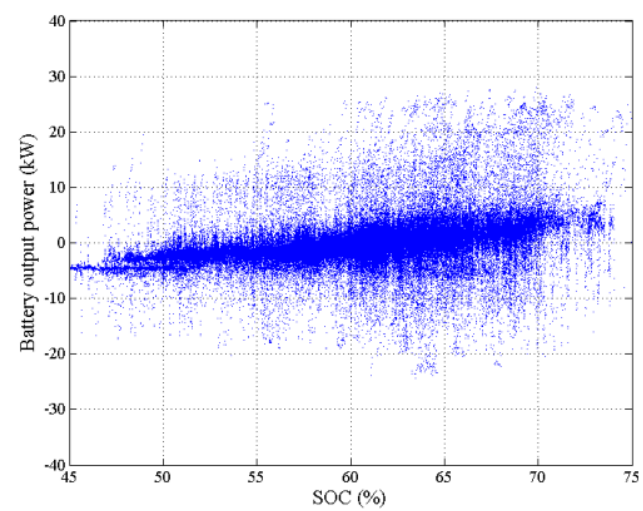

Figure 7. The battery's desired power as determined by the SOC level 


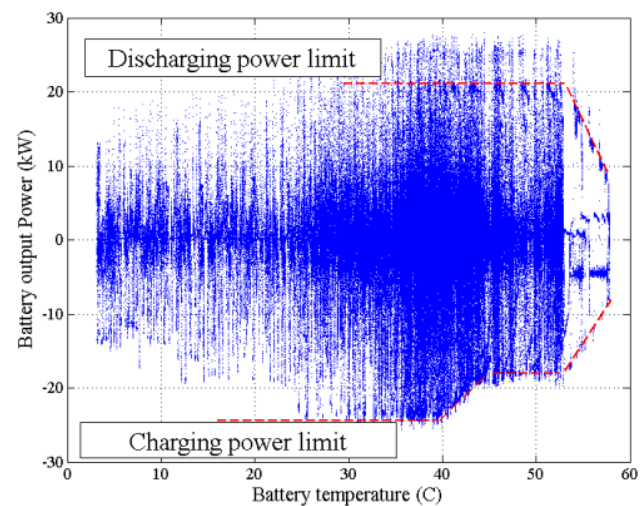

Figure 8 . The output power of the battery as limited by the battery temperature

The control model for each vehicle is developed based on the analysis results, so that the vehicle model in the simulation can provide the same control behavior as the real world vehicle does.

\section{Thermal Model Development}

The thermal components in the powertrain components can be coupled to each other as shown in Figure 9, so it is not easy to develop generic configurations for the thermal management system.

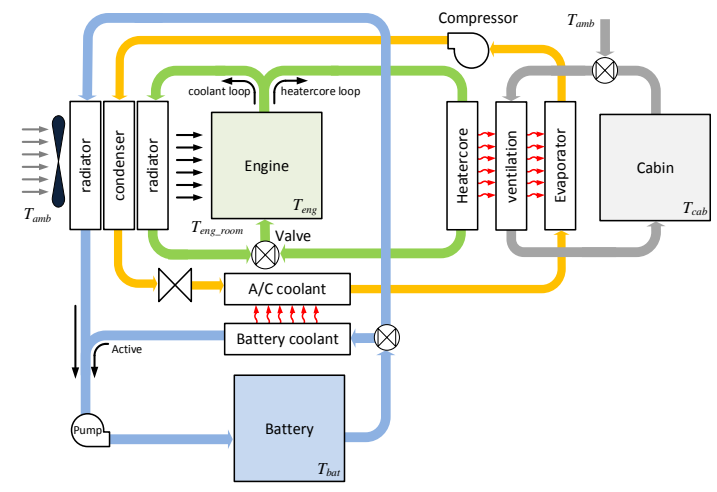

Figure 9. An example of the thermal management system

The thermal management system is thus developed for each vehicle, while there is also an effort to develop generic models and to reuse the thermal component models as much as possible.

\subsection{Thermal Component Model}

This brief introduces the fundamental ideas for developing the powertrain thermal component models here.

\subsubsection{Engine Thermal Model}

The engine thermal components have heat generation and rejection models, as shown in Figure 10.

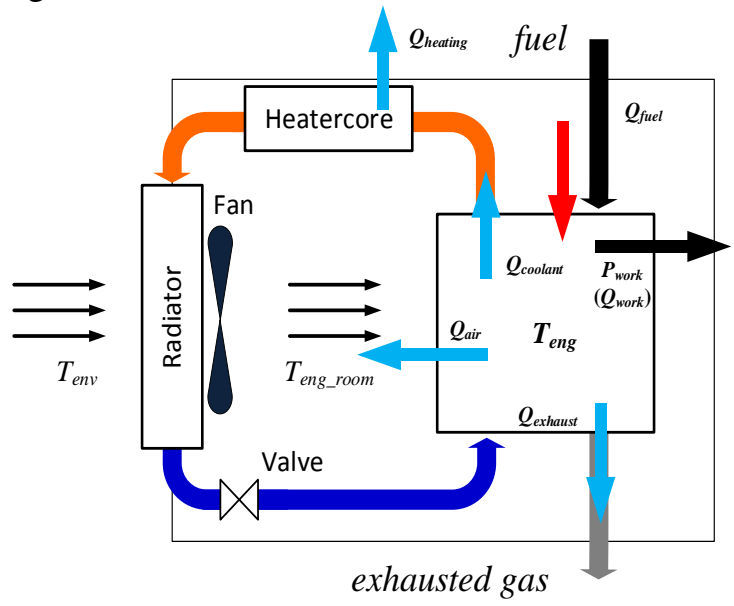

Figure 10. A schematic of the engine thermal model

The heat equation for the engine can be expressed as:

$$
\begin{aligned}
m_{\text {eng }} C_{\text {eng }} \dot{T}_{\text {eng }}= & Q_{\text {fuel }}-P_{\text {work }}-Q_{\text {exhaust }}- \\
& Q_{\text {coolant }}-Q_{\text {air }}-Q_{\text {heating }}
\end{aligned}
$$

where $T_{\text {eng }}, C_{\text {eng }}$, and $m_{\text {eng }}$ are the temperature, the specific heat capacity, and the mass of the engine, respectively. $Q_{\text {fuel }}$ and $P_{\text {work }}$ are the heat generated from the combustion process and the work converted to the mechanical system. $Q_{\text {exhaust }}$, $Q_{\text {coolant }}$, and $Q_{\text {heating }}$ are the heat rejected by the emission gas, the coolant system, and the heat core, respectively. $Q_{\text {air }}$ is the heat transferred to the air by the convection effect.

\subsubsection{Transmission Thermal Model}

An example of the thermal component model for transmission is shown in Figure 11, which is for a Toyota Prius HEV.

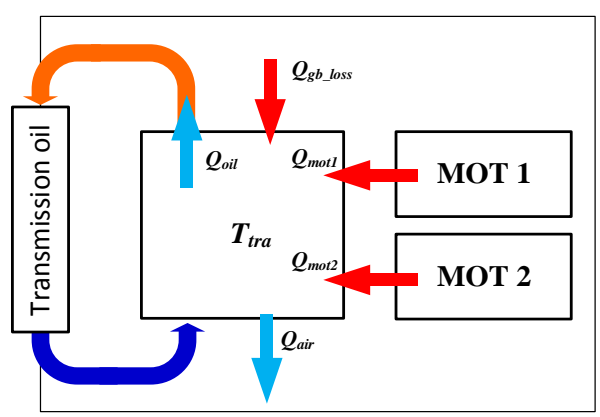

Figure 11. Transmission thermal model 
In the figure, the heat generated by motors, $Q_{\operatorname{mot} 1}$ and $Q_{m o t 2}$, and the loss by the gearbox, $Q_{g b_{-} l o s s}$, are considered for heat sources. The heat rejected by the transmission oil loop and the convection heat transfer between the transmission and the air, $Q_{\text {oil }}$ and $Q_{\text {air }}$, are also considered as:

$$
\begin{aligned}
m_{\text {tra }} C_{\text {tra }} \dot{T}_{\text {tra }}= & Q_{\text {mot } 1}+Q_{\text {mot } 2}+Q_{\text {gb_loss }}- \\
& Q_{\text {oil }}-Q_{\text {air }}
\end{aligned}
$$

where $T_{\text {tra }}, C_{\text {tra }}$, and $m_{\text {tra }}$ are the temperature, the specific heat capacity, and the mass of the transmission, respectively.

\subsubsection{Cabin and Climate Control System}

The climate control system (including the air conditioning system) and the heat balance of the cabin can be expressed as shown in Figure 12.

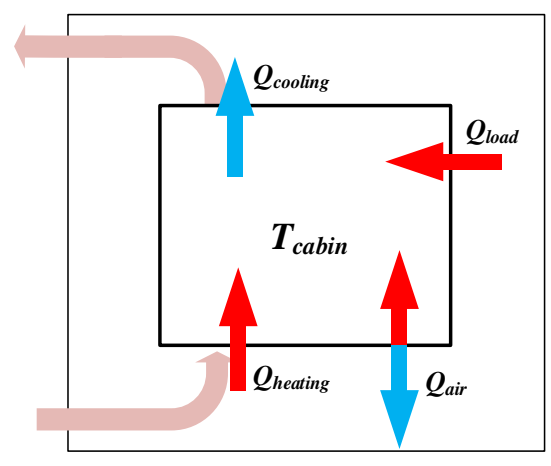

Figure 12. Cabin and climate control system with heat load

The cabin temperature can be calculated as:

$m_{c a b} C_{c a b} \dot{T}_{c a b}=Q_{l o a d}+Q_{h v a c}-Q_{a i r}$

where $T_{c a b}, C_{c a b}$, and $m_{c a b}$ are the temperature, the specific heat capacity, and the mass of the cabin air, respectively. $Q_{\text {hvac }}$ is the heat controlled by the climate control system, and $Q_{\text {air }}$ is the heat convectively transferred between the cabin system and the ambient air. Further, the heat load, $Q_{\text {load }}$, includes the solar heat load, passenger heat load, and heat transferred from powertrain components.

\subsubsection{Battery Thermal Model}

The battery model has the heat source and heat rejection model as well (Figure 13). The heat is generated by the electrochemical and electrical loss, which is calculated by the voltage drop and the output current.

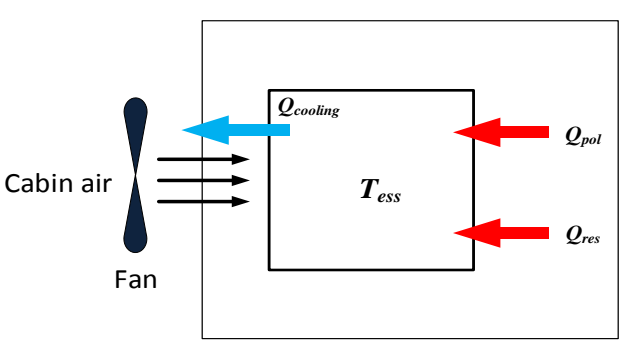

Figure 13. Battery thermal model

The equation for the battery temperature can be expressed as:

$m_{\text {ess }} C_{\text {ess }} \dot{T}_{\text {ess }}=Q_{\text {pol }}+Q_{\text {res }}-Q_{\text {cooling }}$

where $T_{\text {ess }}, C_{\text {ess }}$, and $m_{\text {ess }}$ are the temperature, the specific heat capacity, and the mass of the battery, respectively. The two heat sources terms, $Q_{p o l}$ and $Q_{\text {res }}$, are calculated from PNGV model with parameters estimated from the test data [11].

\subsection{Control Model}

Figure 14 shows an example of a control model, which is for a Toyota Prius PHEV.

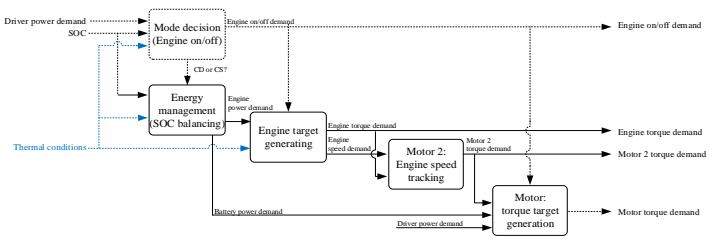

Figure 14. Control flows for Prius PHEV (The decision for $\mathrm{CD}$ or CS mode is added in the engine on/off control; compare this to the control of the HEV.)

In the example, the controller determines the operating mode - like charge depleting (CD) or charge sustaining (CS) - according to the SOC, and it turns on the engine if the demand power exceeds the threshold power. Moreover, the thermal conditions, such as the engine coolant temperature or the battery temperature, also affect the control flow, as shown in Figure 14. Each vehicle model should have its own controller to properly operate the powertrain components, and the controller should reproduce the real world control behaviors well.

\section{Vehicle System and Validation}

The vehicle model can be completed by integrating all the powertrain components and the controller into the system model. Figure 15 shows an example for a Toyota Prius HEV. 


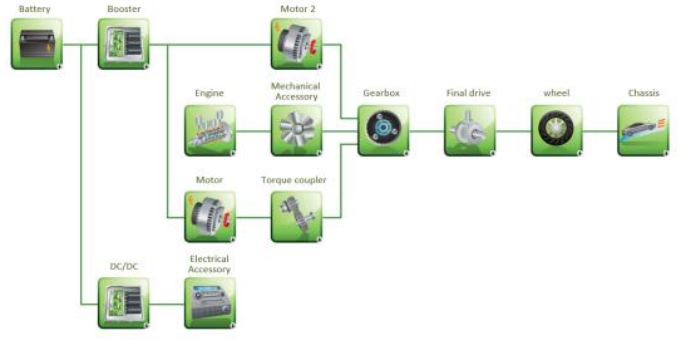

Figure 15. Vehicle configuration of 2010 Toyota Prius in Autonomie

Autonomie, which was developed by Argonne for vehicle simulations, is used to integrate all the component models, and the vehicle models are validated with the test data by comparing the fuel consumption obtained from the simulation model with the fuel consumption measured in the tests, which are shown in Figure 16.

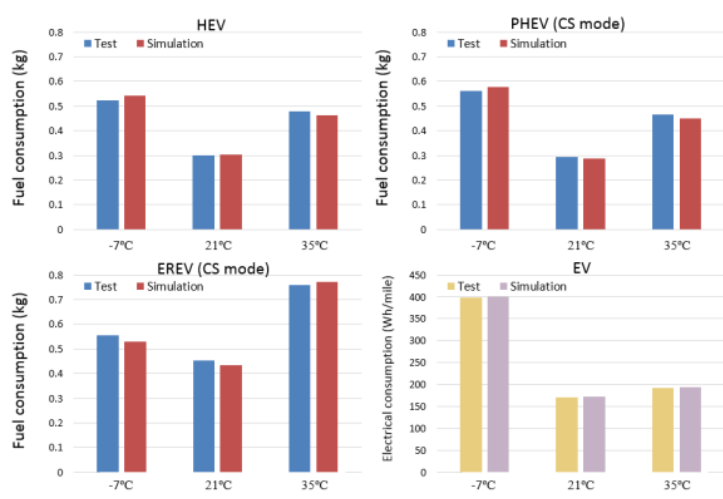

Figure 16. Validation results for four vehicle configurations

The comparison results show that the simulation models are able to represent the impact of the thermal conditions on the fuel consumption across the vehicle configurations quite well. Based on the comparison, it can be said that the simulation techniques are appropriate for evaluating the vehicle's fuel consumption according to different thermal conditions.

\section{Conclusion}

The main goal of this brief is to describe the entire process for developing thermal management systems for advanced vehicles. First, vehicles should be tested under well-organized test procedures, so that the impact of the different thermal conditions on the vehicle's performance can be seen. Second, the test data should be appropriately analyzed to elicit an understanding of the performance of the powertrain components and the vehicle's control behaviors. Third, simulation models should be developed based on the analyzed results and be well validated with the test results. Argonne National Laboratory, by following these processes, developed several configurations for advanced vehicles, and the validation results show that the simulation models appropriately calculate the fuel consumption of the vehicles under different thermal conditions. The developed vehicles can be used in many other studies, so that the impact of thermal conditions can be analyzed in various types of studies.

\section{Acknowledgments}

This work was supported by the U.S. Department of Energy's Vehicle Technology Office under the direction of David Anderson and Lee Slezak. The submitted manuscript has been created by UChicago Argonne, LLC, Operator of Argonne National Laboratory ("Argonne"). Argonne, a U.S. Department of Energy Office of Science laboratory, is operated under Contract No. DE-AC02-06CH11357. The U.S. Government retains for itself, and others acting on its behalf, a paid-up, nonexclusive, irrevocable, worldwide license in said article to reproduce, prepare derivative works, distribute copies to the public, and perform publicly and display publicly, by or on behalf of the Government.

\section{References}

[1] Kim, N., A. Rousseau, D. Lee, and H. Lohse-Busch, "Thermal Model Development and Validation for 2010 Toyota Prius," SAE Technical Paper 2014-011784, 2014.

[2] Kim, N., E. Rask, and A. Rousseau, "Control Analysis under Different Driving Conditions for Peugeot 3008 Hybrid 4," SAE Int. J. Alt. Power. 3(1):44-55, 2014.

[3] Lohse-Busch, H., M. Duoba, E. Rask, K. Stutenberg, et al., "Ambient Temperature $\left(20^{\circ} \mathrm{F}, 72^{\circ} \mathrm{F}\right.$ and $\left.95^{\circ} \mathrm{F}\right)$ Impact on Fuel and Energy Consumption for Several Conventional Vehicles, Hybrid and Plug-In Hybrid Electric Vehicles and Battery Electric Vehicles," SAE Technical Paper 2013-01-1462, 2013.

[4] Rousseau, A., J. Kwon, P. Sharer, S. Pagerit, S., et al., "Integrating Data, Performing Quality Assurance, and Validating the Vehicle Model for the 2004 Prius Using PSAT," SAE Technical Paper 2006-01-0667, 2006.

[5] Cao, Q., S. Pagerit, R.B. Carlson, and A. Rousseau, "PHEV Hymotion Prius Model Validation and Control Improvements," in Proc. EVS23, Anaheim, CA, 2007.

[6] Kim, N., A. Rousseau, and E. Rask, "Vehicle-Level Control Analysis of 2010 Toyota Prius based on Test 
Data," IMechE Part D: J. Automobile Engineering, vol. 226, no. 11, pp. 1483-1494, Nov. 2012.

[7] Jehlik F., and E. Rask, "Development of Variable Temperature Brake Specific Fuel Consumption Engine Maps," SAE Technical Paper 2010-012181, 2010.

[8] Jehlik, F., E. Rask, and M. Christenson, "Simplified Methodology for Modeling Cold Temperature Effects on Engine Efficiency for Hybrid and Plugin Hybrid Vehicles," SAE Technical Paper 201001-2213, 2010

[9] Information available at http://www.transportation. anl.gov/facilities/4WD_testcell.html

[10] Data available at http://www.transportation.anl.go v/D3/2010_toyota_prius.html

[11] Hu, X., S. Li, and H. Peng, “A Comparative Study of Equivalent Circuit Models for Li-ion Batteries," Journal of Power Sources, Vol. 198, No. 1, pp. 359-367, 2012.

\section{Authors}

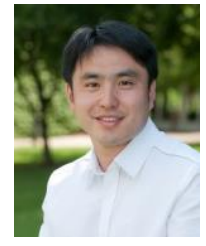

Namwook Kim received his B.S. degree in 2003 and $\mathrm{Ph} . \mathrm{D}$ in 2009 from Seoul National University. He joined Argonne National Laboratory in 2009 as a postdoc and is current working as a research engineer for transportation research center at the laboratory.

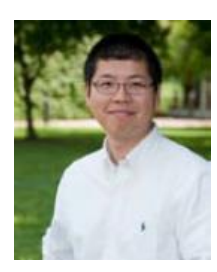

Namdoo Kim graduated in 2007

from the University of Sungkyunkwan, Korea, with a Master's degree in School of Mechanical Engineering. $\mathrm{He}$ is currently working in Argonne National Laboratory's Vehicle Modeling and Simulation group.

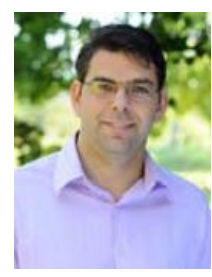

Aymeric Rousseau received his Master of Science in Industrial Systems from EIGSI in La Rochelle, France, in 1997. He is currently leading Argonne's Vehicle modeling and simulation group. 